\title{
Mixoma de átrio direito com prolapso para o ventrículo direito
}

\author{
Right atrial myxoma prolapsing into the right ventricle
}

José Glauco LOBO FILHO ${ }^{1}$, Dadson Leandro de Sá SALES², Allison Emídio Pinheiro Pereira BORGES², Maria Cláudia LEITÃO ${ }^{3}$

\section{Resumo}

Relatamos o caso raro de uma paciente de 67 anos, apresentando mixoma de átrio direito, com prolapso para o ventrículo direito. Estes tumores correspondem a cerca de $18 \%$ dos casos de mixoma, que por sua vez têm uma incidência de $0,0017 \%$ na população em geral.
Descritores: Mixoma. Neoplasias cardíacas. Átrios do coração.

\section{Abstract}

We report on a rare case of a 67 -year-old woman with a right atrial myxoma prolapsing into the right ventricle in the diastolic phase. These tumors comprise approximately $18 \%$

1 - Doutor em cirurgia cardíaca. Cirurgião cardiovascular e diretor da Divisão de Cardiologia do Hospital Regional Unimed - Fortaleza, CE.

2 - Estudante (Interno) do curso de Medicina da Universidade Federal do Ceará. Estagiário bolsista do Instituto do Coração e Pulmão (ICORP) - Fortaleza, CE.

3 - Especialista em Cardiologia e Ecocardiografia pela Sociedade Brasileira de Cardiologia. Cardiologista e Ecocardiografista do Instituto do Coração e Pulmão (ICORP). Cardiologista do Hospital do Coração, Fortaleza, CE.

Trabalho realizado no Hospital Regional Unimed, Fortaleza, CE.

Endereço para correspondência:

José Glauco Lobo Filho. Rua Dr. José Lourenço, 625. Aldeota. Fortaleza, CE, Brasil. CEP: 60115-280. Tel: (85) 3244-1711 Fax: (85) 3261-0816.

E-mail: glaucolobo@uol.com.br; dadson@superig.com.br 


\section{INTRODUÇÃO}

O mixoma cardíaco é uma neoplasia benigna, que corresponde ao tumor primário mais comum do coração, respondendo por cerca de $50 \%$ destes [1]. De modo geral, 75 a $80 \%$ dos mixomas estão localizados no átrio esquerdo, $18 \%$ no átrio direito, e mais raramente, nos ventrículos ou multicêntricos [2]. Os pacientes apresentam pelo menos uma das características de uma tríade classicamente descrita, que inclui: sintomas de obstrução cardíaca e constitucionais, além de eventos embólicos [3]. Podem, ainda, apresentar arritmias cardíacas, devido tanto à infiltração direta do tecido de condução cardíaco, como à irritação do próprio miocárdio. O mixoma atrial direito, em particular, pode obstruir a valva tricúspide, causando sintomas de insuficiência cardíaca direita, edema periférico, congestão hepática e síncope [3].

Nosso objetivo é relatar um caso de mixoma de átrio direito com prolapso para o ventrículo direito (VD) através da valva tricúspide.

\section{RELATO DO CASO}

Paciente do sexo feminino, 67 anos, branca, foi admitida em nosso serviço com queixa de fadiga e adinamia há cerca de três meses. Relata, no período, queixas de dispnéia leve que melhorava com o repouso, tosse não produtiva, sem hemoptise, e dois episódios de pletora cervical, que regrediram espontaneamente. Há cerca de 45 dias, relata dois episódios de febre leve, mensurada em $38^{\circ} \mathrm{C}$, sem etiologia definida e que a paciente atribuiu a um estado gripal. Relata, ainda, história de asma, hipertensão arterial sistêmica (HAS) controlada com o uso de enalapril $20 \mathrm{mg} / \mathrm{dia}$ e mastectomia total à direita realizada há cinco anos para tratamento de câncer de mama, associada à quimioterapia e radioterapia. Ao exame físico, a freqüência cardíaca era de 100 batimentos por minuto, com pressão arterial de 130 por $80 \mathrm{mmHg}$. Encontrava-se afebril, sem cianose central ou periférica. Apresentava-se com ausculta cardiopulmonar fisiológica e sem massas ou visceromegalias à palpação abdominal.

A avaliação laboratorial hematológica não apresentava anormalidades, exceto por um aumento na velocidade de hemossedimentação (VHS) e da Proteína-C Reativa, com valores de $114 \mathrm{~mm}$ e $6,47 \mathrm{mg} / \mathrm{dl}$, respectivamente. Todos os outros exames laboratoriais de rotina estavam dentro dos limites da normalidade. O eletrocardiograma pré-operatório não apresentava alterações. A radiografia de tórax mostrava um aumento da silhueta cardiovascular e velamento da cúpula diafragmática esquerda.

Foi realizada uma tomografia computadorizada (TC) de tórax com contraste venoso, que revelou uma pequena efusão pleural esquerda, derrame pericárdico com espessura de dois milímetros, mastectomia direita e aparente falha de enchimento de átrio direito. Foram realizadas ainda TC de crânio, que se mostrou sem alterações e TC de abdome e pelve, mostrando cisto cortical renal direito (Bosniak I), pequeno nódulo esplênico e ateromatose aórtica. Ecocardiograma transtorácico revelou uma massa medindo aproximadamente $2,5 \times 5,5 \mathrm{~cm}$ inserida no átrio direito, que se movia para o interior do VD na diástole, através da valva tricúspide e movimento paradoxal do septo atrial, compatível com mixoma de átrio direito (Figura 1). A coronariografia para avaliação de doença coronariana não evidenciou anormalidades.

Com a referida hipótese diagnóstica, a paciente foi

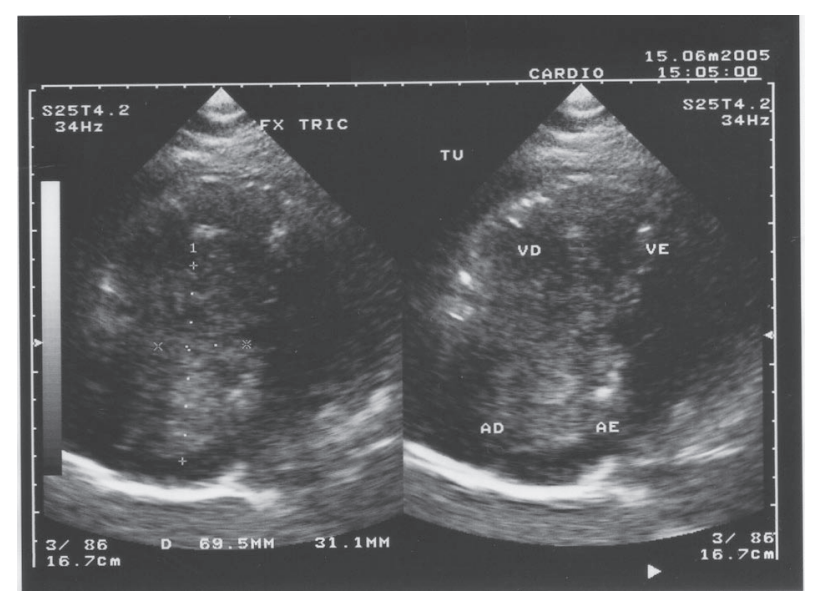

Fig. 1 - Ecocardiograma transtorácico mostrando uma massa medindo aproximadamente $2,5 \times 5,5 \mathrm{~cm}$, inserida no átrio direito.

encaminhada ao serviço de cirurgia cardíaca, sendo, então, submetida à exérese do referido tumor, utilizandose a técnica a seguir: 1. Mediastinotomia transesternal longitudinal; 2. Pericardiotomia longitudinal, observandose aumento acentuado do átrio direito com demais estruturas cardíacas normais; 3 . Instalação de circuito de circulação extracorpórea (CEC), utilizando-se oxigenador de membrana Braile Biomédica; 4. Anticoagulação realizada com 400UI/kg de peso de heparina sódica; 5.A CEC foi instituída por meio de cânula arterial posicionada no final da aorta ascendente. A drenagem da veia cava inferior se fez pelo posicionamento de cânula venosa, com bolsa realizada no átrio direito, um centímetro acima da emergência da mesma. A drenagem da veia cava superior se fez por meio do posicionamento de cânula venosa na mesma, com bolsa realizada um centímetro abaixo de sua emergência no átrio direito; 6. Após pinçamento aórtico, realizou-se proteção miocárdica anterógrada com sangue oxigenado isotérmico e hipercalêmico; 7. Após a parada cardíaca, com o sangue desviado integralmente do átrio 
direito, realizou-se a atriotomia direita, visibilizando-se uma volumosa massa tumoral com bordas sem aderências às estruturas cardíacas (Figura 2), porém com sua base pediculada inserida no septo interatrial, próximo ao resquício do forame oval; 8. A exérese do referido tumor se fez pela retirada de toda a área septal relativa à implantação do mesmo, com posterior septoplastia, utilizando-se remendo de pericárdio bovino; 9. Após fechamento do átrio direito, retirada de ar das cavidades de maneira usual e restituição da circulação pulmonar e coronariana, o coração voltou ao ritmo sinusal; 10. Com a normalização da temperatura corporal, a CEC foi descontinuada, procedendo-se, após a retirada das cânulas, à neutralização da heparina com a administração de cloridrato de protamina na dose de $1 \mathrm{mg}$ para cada 100UI de heparina utilizada na anticoagulação. Após o término da cirurgia, constatou-se que o tumor media aproximadamente $2,5 \times 5,0 \mathrm{~cm}$, sendo encaminhado para estudo histopatológico, que ratificou o diagnóstico de mixoma. $\mathrm{O}$ curso pós-operatório imediato transcorreu sem intercorrências, permanecendo a paciente dois dias na UTI pós-operatória, com alta hospitalar no $7^{\circ}$ dia, sem evidências de sinais ou sintomas de insuficiência cardíaca.

\section{DISCUSSÃO}

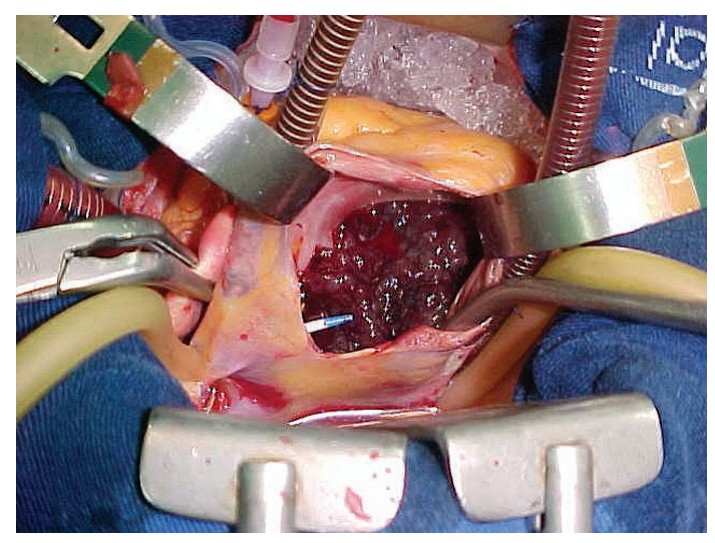

Fig. 2 - Mixoma de átrio direito - Visibilização de volumosa massa tumoral após atriotomia direita.

Tumores primários do coração são entidades raras, ocorrendo em 0,0017\% em séries de autópsias [4]. Destes, o mixoma cardíaco é o mais comum, sendo uma afecção que acomete pacientes dentro de uma larga faixa etária (15-80 anos), com uma média aproximada de cinqüenta anos. Parece haver uma ligeira predominância feminina, com uma razão de mulheres em relação a homens de 5:4
[3]. Apesar de apresentarem caráter histológico benigno, podem levar a evolução desfavorável, sendo responsáveis por complicações incapacitantes [1].

Devido a uma apresentação clínica não específica dos pacientes portadores de mixoma, o diagnóstico pré mortem só foi conseguido em 1952, quando Goldberg demonstrou um mixoma de átrio esquerdo por meio de uma angiografia cardíaca [3]. A primeira ressecção cirúrgica, com êxito, de um mixoma cardíaco foi realizada, com auxílio de CEC, em 1954 e este paciente continuava vivo até 1997 [3].

O sintoma mais comum relacionado ao mixoma cardíaco é a insuficiência cardíaca congestiva, seguido por embolização, seja esta sistêmica ou pulmonar [5]. Estes sintomas, geralmente relacionados com a localização dos tumores, variam com seu tamanho e forma, além da atividade física e da posição do paciente. Com relação ao mixoma de átrio direito, a apresentação clínica pode incluir ascite, hepatomegalia ou edema periférico, todos devido à insuficiência cardíaca direita. Sintomas constitucionais vagos podem também ser encontrados, como mal estar, febre geralmente baixa ou de longa duração e perda de peso [5]. Estes sintomas, assim como anemia, aumento de VHS e dos níveis séricos de gamaglobulina podem causar confusão no diagnóstico [6], pois são decorrentes de uma resposta inflamatória, passíveis de serem encontradas em diversas doenças. Nesse caso de mixoma atrial direito, a paciente apresentava dispnéia aos esforços, tosse não produtiva, episódios de congestão cervical e febre baixa $\left(38^{\circ} \mathrm{C}\right)$.

Embora o ecocardiograma transtorácico seja menos invasivo e apresente uma excelente sensibilidade de $95 \%$ na detecção de mixomas, a sensibilidade diagnóstica pode atingir $100 \%$, quando sucedido de uma ecocardiografia transesofágica. A TC e a ressonância magnética podem ser úteis ao demonstrarem o ponto de fixação e complicações associadas. O recente progresso nas modalidades diagnósticas em cardiologia, incluindo os exames supracitados, tem permitido realizar o diagnóstico de tumores cardíacos primários sem o uso de cateterismo cardíaco ou angiografia, precisando, de forma confiável, a real localização do tumor.

Desse modo, a canulação das veias cavas, para a instalação da CEC, pode ser feita com segurança através do átrio direito, observando-se os cuidados técnicos, a fim de evitar o desprendimento de massa tumoral com conseqüente embolização, principalmente nos casos em que haja defeito no septo interatrial. Evita-se, assim, a canulação da veia femoral, minimizando-se as complicações cirúrgicas.

A freqüência de recorrência de mixomas cardíacos varia de $3 \%$ nos casos esporádicos, como o aqui relatado, a $22 \%$ nos casos associados ao "complexo de Carney" [4]. 


\section{REFERÊNCIAS}

1. Mota AAR, Colen Filho E, Colen EA, Vieira JAS, Alves MAP, Borges MF et al. Mixoma do átrio esquerdo: relato de 3 casos. Rev Bras Cir Cardiovasc. 1997;12(4):377-83.

2. Ipek G, Erentug V, Bozbuga N, Polat A, Guler M, Kirali K et al. Surgical management of cardiac myxoma. J Card Surg. 2005;20(3):300-4.

3. Grebenc ML, Rosado-de-Christenson ML, Green CE, Burke AP, Galvin JR. Cardiac myxoma: imaging features in 83 patients. Radiographics. 2002;22(3):673-89.
4. Acebo E, Val-Bernal JF, Gomez-Román JJ, Revuelta JM. Clinicopathologic study and DNA analysis of 37 cardiac myxomas: a 28-year experience. Chest. 2003;123(5):137985.

5. Murayama H, Tamaki S, Kato N, Yuji N, Yokote J, Mutsuga $\mathrm{M}$ et al. Right atrial myxoma associated with atrial septal defect: a case report and review of the literature. Ann Thorac Cardiovasc Surg. 2001;7(3):166-9.

6. Karachalios G, Bablekos G, Karachaliou I, Zoganas L, Charalabopoulos A, Charalabopoulos K. Left atrial myxoma prolapsing into the left ventricle: case report and review of the 